\title{
Amphiphilic Co-network Based on the Cross-Linked Star Copolymers Designed for Efficient Antibiofouling Coating
}

\author{
Haiye Wang ${ }^{a}$, Dapeng Liu ${ }^{b}$, Chengfeng Zhang ${ }^{c}$, Chunju He ${ }^{d}$ \\ College of Materials Science and Engineering, Donghua University, Shanghai, 201620, P. R. China \\ a615434312@qq.com, ${ }^{\mathrm{b}} \mathrm{dp} 1573 @ 163 . c o m,{ }^{\mathrm{c}}$ iriscfz@163.com, ${ }^{\mathrm{d}}$ chunjuhe@dhu.edu.cn
}

Keywords: star copolymers, amphiphilic conetworks, antibiofouling coating.

\begin{abstract}
Synthesis of binary amphiphilic co-networks coatings composed of well-defined tri-arm star diblock copolymers cross-linked with polyhydrosiloxanes through hydrosilylation was performed to generate antifouling surfaces. The PMHS segments of the networks endow the coating with a low surface energy while the $\mathrm{N}$-isopropylacrylamide (DMAAm) was added to weaken fouling adhesion. Antifouling characteristics were investigated by bovine serum albumin (BSA) adsorption studies; the coating displayed a $60 \%$ greater resistance to protein adsorption in comparison to the fouling of a control. The unique surface topography and chemical heterogeneity expressed at a variety of scales generate complex surfaces known to incorporate characteristics appropriate for antifouling applications. The nontoxicity in relevant environment demonstrated it appropriate for consideration in marine applications.
\end{abstract}

\section{Introduction}

Amphiphilic co-networks (APCN) based on the covalently interconnected of hydrophilic and hydrophobic polymer segments belong to a novel family of cross-linked polymeric materials.[1] The immiscibility of the hydrophilic and hydrophobic components leads to phase separation at the nanoscale,[2,3] which renders APCNs a broad potential application. Recently, they have been increasingly evaluated as environmentally friendly antifouling coatings with a utility for biofouling prevention in marine fields.[4]

It is well-known that star block copolymers exhibit different properties in comparison with their linear counterparts due to their well-defined branched structure, high solubility in various solvents, low melt viscosity, and controlled functionality.[5] Consequently, the APCNs prepared from amphiphilic polymer with star-shaped structures have also attracted researchers' attention.

In this study, a hydrophilic star homepolymer was synthesized via ATRP to produce the allyl precursor for the preparation of end-linked amphiphilic conetworks. Nonspecific protein resistance studies were constructed using BSA as the model protein. The special surface composition, amphiphilic character, as well as the protein resistance of the prepared APCN indicate a promising antifouling materials in marine fields.

\section{Experimental part}

\subsection{Materials.}

Reagents and starting materials were purchased from Energy Chemical Co. and used as received unless otherwise noted. $\mathrm{N}$-isopropylacrylamide (DMAAm) were purchased from Aldrich and dried from calcium chloride prior to use. Glass slides were purchased from Fisher and pretreated according to the literature.[6] Three-arm star initiator was synthesized according to our previous work.[5]

\subsection{Synthesis of three-arm star PDMAAm copolymer.}

Three-arm star initiator, DMAAm and cyclohexanone were added to a Schlenk flask. $\mathrm{CuBr}$ was added under Ar and then the flask was heated at $60{ }^{\circ} \mathrm{C}$ for $12 \mathrm{~h}$. The crude product was dialyzed against distilled water over 3 days, prior to being dried in vacuum for $24 \mathrm{~h}$. 


\subsection{Synthesis of three-arm star-allyl PDMAAm copolymer.}

The three-arm star-allyl PDMAAm copolymer was synthesized using a molar feed ratio [star PDMAAm]:[KOH]:[Ally amine] of $1: 1: 150$ in $20 \mathrm{~mL}$ DMF at $50{ }^{\circ} \mathrm{C}$ for $24 \mathrm{~h}$. The solution was dialyzed against distilled water for 7 day. The product was finally freeze-dried.

\subsection{Synthesis of the amphiphilic coatings on microscope glass slides.}

The pretreated slides were coated by multiple cycles of immersion into a toluene solution containing star-allyl PDMAAm homopolymer $(1 \mathrm{~g})$, PMHS $(0.6 \mathrm{~g})$ and Karstedt's catalyst $(0.05 \mathrm{~mL})$. The glass slides were then heated at $100{ }^{\circ} \mathrm{C}$ for $1 \mathrm{~h}$ under nitrogen. In each case, all the coating were slightly yellow upon curing.

Characterization. 1H NMR spectra was performed at room temperature on a Bruker Avance 400 instrument using $\mathrm{CDCl}_{3}$ solutions with tetramethylsilane (TMS) as an internal standard. FT-IR spectra were obtained with a Nicolet Instrument Nicolet 8700 spectrometer using a $\mathrm{KBr}$ disk. The adsorption of FITC-BSA on the coating was observed with an Olympus BX-51 inverted microscope and an Olympus DP52 digital camera.

\section{Results and discussion}

\subsection{Synthesis Methodology.}

Fig.1 illustrates the synthetic strategy of the amphiphilic co-network (APCN) coating on the substrate. The first step is the synthesis of three-arm star initiator by coupling triethanolamine with 2-bromoisobutyryl bromide. Here, star-PDMAAm was synthesized via ATRP of DMAAm using three-arm star initiator and $\mathrm{CuBr} / \mathrm{PMDETA}$ catalyst system. Then, end bromine of the star-PDMAAm was then transformed to an active allyl group through nucleophilic substitution. The present research concerns the synthesis of APCN by combining star-allyl PDMAAm with polymethylhydrosiloxane (PMHS) through hydrosilylation. There exists an average of $100 \mathrm{Si}-\mathrm{H}$ pendant bonds per PMHS chain available for hydrosilylation. Variations in the relative amounts of star-allyl PDMAAm and PMHS could result in differences in the crosslinking densities and also in the overall compositions of the network composite materials. The stoichiometry of the different components is an important parameter for tuning the surface features, and the thermodynamically driven phase segregation of star-allyl PDMAAm from PMHS is kinetically trapped by the crosslinking reactions. In this article, this process of thermodynamic assembly and kinetic crosslinking serves as a general methodology for improving the surface antifouling properties of the coatings.

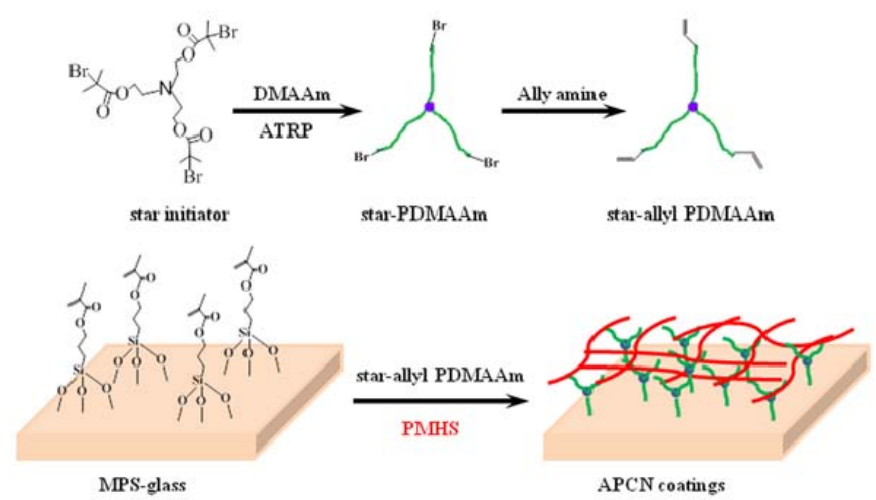

Fig.1 Schematic illustration for the preparation of cured star-allyl PDMAAm/PMHS coating on substrate.

\subsection{Characterization of the star copolymer.}

The compositions of the network precursors were determined by ${ }^{1} \mathrm{H}$ NMR spectroscopy. Figure 1 (a) shows the ${ }^{1} \mathrm{H}$ NMR spectroscopy of the star PDMAAm copolymer. The chemical shift at 2.05 ppm is assigned to the protons $\left(\mathrm{d}, \mathrm{BrC}\left(\mathrm{CH}_{3}\right)_{2}\right)$ of the 2-bromoisobutyryl groups. The signals at 1.4, 1.7 and $3.0 \mathrm{ppm}$ are mainly assigned to the methylene $\left(\mathrm{c}, \mathrm{CH}_{2}\right)$, methylidyne $(\mathrm{b}, \mathrm{CH})$ and methyl $(\mathrm{a}$, $\left.\mathrm{N}\left(\mathrm{CH}_{3}\right)_{2}\right)$ protons of the DMAAm units. Figure 1 (b) shows the ${ }^{1} \mathrm{H}$ NMR spectra of star-allyl 
PDMAAm. The chemical shifts at 5-6 ppm correspond to the protons of allyl group units, indicating the successful modification of the star-allyl PDMAAm.
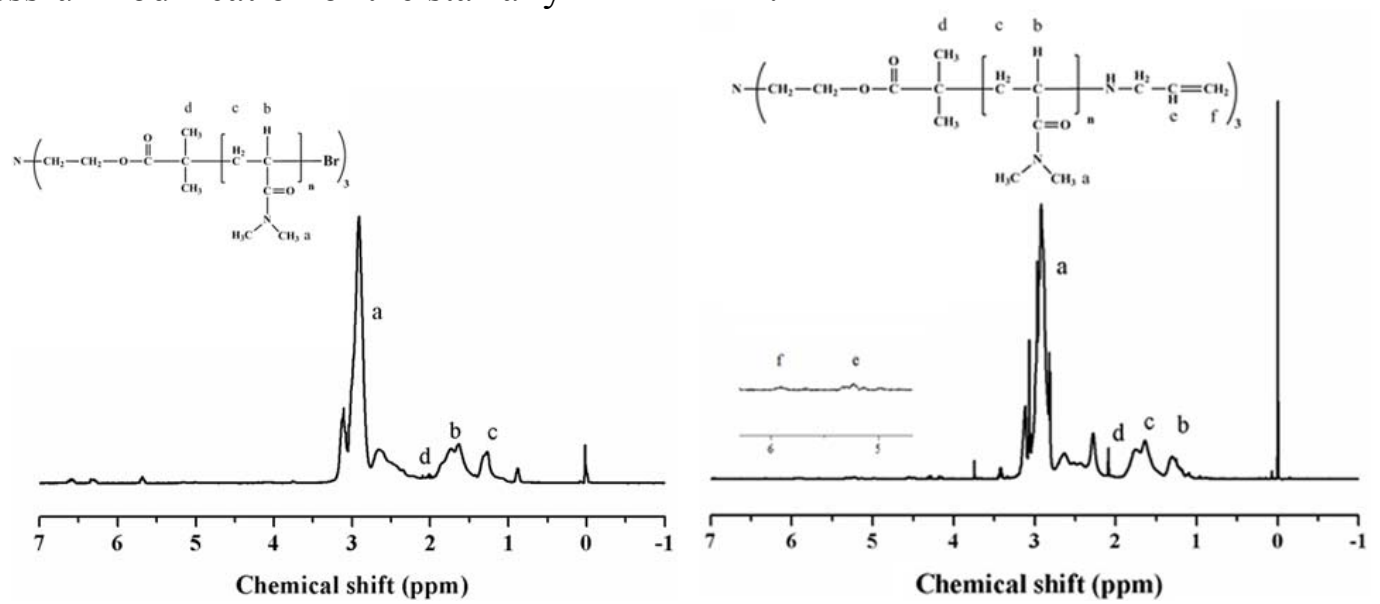

Fig.2 The 1H NMR spectra of the (a) star-PDMAAm; (b) star-allyl PDMAAm;

Table 1 Characterization data of the cross-linked coatings

\begin{tabular}{ccc}
\hline \multicolumn{3}{c}{ Coating composition } \\
\hline Coating & star-allyl PDMAAm (wt\%) & PMHS (wt $\%)$ \\
\hline APCN-1 & 15 & 85 \\
\hline APCN-2 & 45 & 55 \\
\hline
\end{tabular}

\subsection{Nonspecific protein resistance.}

As depicted in Fig. 3, uniform and intense fluorescence was observed for the original MPS-glass control, indicating a significant BSA-FITC adsorption on the MPS-glass surface. Compared with the original glass control, the fluorescence intensities of the APCN coatings decreased obviously. With increasing DMAAm contents in the coating surfaces, the protein resistance effect became more significant. The result shows less adsorption and enhanced protein resistance of the materials, indicating that the content of DMAAm in the amphiphilic coating played an important role.

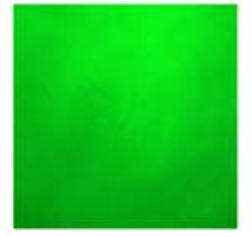

a

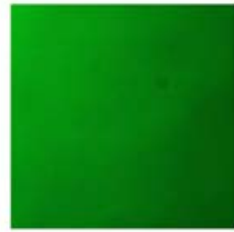

b

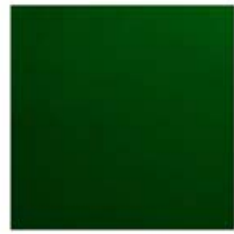

Fig.3 Fluorescence microscopy images of BSA adsorption on the control and coatings.(a) MPS-Glass (b) APCN-1 (c) APCN-2

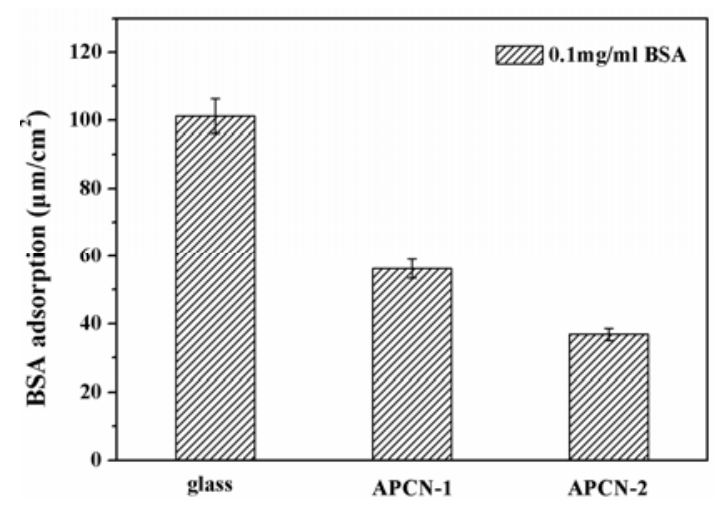

Fig.4 BSA adsorption amounts on MPS-Glass and the APCN coatings. 
In order to quantitatively estimate the protein resistance of the coatings, static BSA adsorption on varied coatings surfaces were investigated. As described in Fig.4, BSA adsorption on the APCN coatings with different compositions is much lower compared with that of the virgin MPS-Glass surface. The amount of adsorbed BSA decreased significantly with increasing content of the PDMAAm chain in the coatings. Compared with adsorption of the control glass, APCN-2 displayed a $60 \%$ greater resistance to protein adsorption. The significant decreasing of the BSA adsorption could be attributed to the distribution of hydrophilic DMAAm chains on the APCN surfaces with a repellent property for protein adsorption. On the other hand, the unique surface topography and chemical heterogeneity also generate a phase-segregated complex surfaces, which would be improving the antifouling property. The research result shows that the APCN surface with a designed structure and composition could provide an efficient protein resistance.

\section{Summary}

Novel amphiphilic conetworks coating was successfully fabricated through in-one-step end-cross-linking of well-defined allyl ditelechelic star diblock copolymers via ATRP. The prepared coating exhibited composition heterogeneity and amphiphilic character. With increasing DMAAm content in the coatings, the fouling resistance to the protein BSA was enhanced, which was resulted from the strong hydration of DMAAm. The APCN-2 achieved a protein-resistant rate of $60 \%$ compared with the blank control. The designed coatings show good prospects in potential applications in marine coating.

\section{References}

[1]. C. S. Patrickios, T. K. Georgiou, et al. Covalent amphiphilic polymer networks. Curr. Opin. Colloid Interface. Vol. 8 (2003), No. 1, p. 76-85.

[2]. G. Kali, T. K. Georgiou, C. S. Patrickios, et al. Synthesis and Characterization of Anionic Amphiphilic Model Conetworks of 2-Butyl-1-Octyl-Methacrylate and Methacrylic Acid: Effects of Polymer Composition and Architecture. Langmuir. Vol. 23 (2007), No. 21, p. 10746-10755.

[3]. G. Kali, T. K. Georgiou, C. S. Patrickios, et al. Anionic Amphiphilic End-Linked Conetworks by the Combination of Quasiliving Carbocationic and Group Transfer Polymerizations. J. Polym. Sci., Part A: Polym. Chem. Vol. 47 (2009), No. 21, p. 4289-4301.

[4]. E. Martinelli, M. Suffredini, G. Galli, et al. Amphiphilic block copolymer/poly (dimethylsiloxane) (PDMS) blends and nanocomposites for improved fouling-release. Biofouling. Vol. 27 (2011), No. 5, p. 529-541.

[5]. H. Wang, A. Qin, C. He, et al. Biocompatible amphiphilic conetwork based on crosslinked star copolymers: A potential drug carrier. J. Polym. Sci., Part A: Polym. Chem. DOI: 10.1002/pola.27721.

[6]. Y. Dong, X. Zhu, J. Nie, et al. Surface photo-anchored PNIPAM crosslinked membrane on glass substrate by covalent bonds. Appl. Surf. Sci. Vol. 307 (2014), p. 7-12. 